\title{
Thermodynamics of Anomaly-Driven Cosmology
}

\author{
James E. Lidsey \\ Astronomy Unit, School of Mathematical Sciences, \\ Queen Mary University of London, London E1 4NS, United Kingdom
}

\begin{abstract}
The Friedmann equations of general relativity can be derived from the first law of thermodynamics when the entropy of the apparent horizon of a spatially isotropic universe is given by the Bekenstein-Hawking entropy. We point out that if the entropy of the apparent horizon receives a logarithmic correction, the first law of thermodynamics leads to a modified Friedmann equation which corresponds precisely to the time-time component of the semi-classical Einstein field equations sourced by the trace anomaly of $\mathcal{N}=4 U(N)$ super-Yang-Mills theory. This correspondence allows for a thermodynamic description of the dynamics of the Randall-Sundrum braneworld scenario.
\end{abstract}

\section{Introduction}

It is well known that the entropy and temperature of the event horizon of a Schwarzschild black hole are given by the Bekenstein-Hawking entropy [1]

$$
S_{\mathrm{BH}}=\frac{A}{4 G}
$$

and the Hawking temperature [2]

$$
T_{H}=\frac{1}{2 \pi \tilde{r}_{A}}
$$

respectively, where $A=4 \pi \tilde{r}_{A}^{2}$ is the proper area of the horizon and $G$ is Newton's constant. Similar expressions arise for the cosmological event horizon of de Sitter space [3]. More generally, if the entropy of a local Rindler causal horizon is proportional to its area, the Einstein field equations can be derived from the fundamental thermodynamic relation $\delta Q=T d S$, where the heat flow, $\delta Q$, is interpreted in terms of the energy flux across the horizon and the temperature is the Unruh temperature associated with a uniformly accelerating observer just within the horizon [4]. Similar ideas have been employed in a cosmological context [5, 6, 7, 8, 9, 10, 11]. It can be shown that if the entropy of the apparent horizon of a spatially isotropic universe is related to its area by Eq. (1), the Friedmann equations of general relativity follow from the first law of thermodynamics [10].

† Email: J.E.Lidsey@qmul.ac.uk 
These developments indicate that there exists a direct correspondence between Einstein gravity and the Bekenstein-Hawking entropy. However, Eq. (11) is a semiclassical relation and becomes modified when quantum corrections are taken into account. One such correction arises when one-loop effects break the conformal invariance of classical massless fields and induce an anomalous trace for the energy-momentum tensor. (For reviews, see, e.g., Refs. [12, 13]). In particular, the entropy of a Schwarzschild black hole that is emitting zero-mass particles receives a logarithmic correction of the form [14, 15, 16]:

$$
S=\frac{A}{4 G}+\alpha \ln \left(\frac{A}{4 G}\right),
$$

where the dimensionless parameter, $\alpha$, is determined by the conformal anomaly of the fields.

In a cosmological context, it was recently shown that a logarithmic correction to the entropy of the apparent horizon generates a modified Friedmann equation when the first law of thermodynamics is applied [17]. On the other hand, the conformal anomaly is purely geometrical in nature and also modifies the dependence of the Friedmann

equation on the energy density of the universe. In view of the above correspondence between gravity and thermodynamics, it is of interest to investigate whether the modifications due to the trace anomaly can be reproduced by a logarithmically-corrected Bekenstein-Hawking entropy. In the present work, we find that the corrections to the spatially flat Friedmann equation derived from the thermodynamic and field-theoretic perspectives are identical when the conformal field theory ( $\mathrm{CFT}$ ) is $\mathcal{N}=4, U(N)$ superYang-Mills theory. As in the black hole background, the logarithmic correction to the entropy is proportional to the conformal anomaly of the fields. In this sense, therefore, the conformal anomaly can be interpreted as a quantum correction to the entropy of the apparent horizon.

The structure of the paper is as follows. We begin in Section 2 with the thermodynamic derivation of the Friedmann equation and establish a direct correspondence with anomaly-driven cosmology in Section 3. We employ this correspondence in Section 4 to present a thermodynamic description of the RandallSundrum braneworld scenario. We conclude with a summary in Section 5.

\section{Thermodynamic Friedmann Equation}

The spatially flat and isotropic Friedmann-Robertson-Walker (FRW) line-element is given by $d s^{2}=h_{a b} d x^{a} d x^{b}+\tilde{r}^{2} d \Omega_{2}^{2}$, where $h_{a b}=\operatorname{diag}\left(-1, a^{2}\right), \tilde{r} \equiv r a(t)$ and $a$ denotes the scale factor. On a constant time hypersurface, the apparent horizon of an observer at $r=0$ is the sphere where orthogonal ingoing, future-directed light-rays have zero expansion. This sphere is defined by the condition $h^{a b} \partial_{a} \tilde{r} \partial_{b} \tilde{r}=0$ and has a radius $\tilde{r}_{A}=r_{A} a=1 / H$ and area $A=4 \pi / H^{2}$, where $H \equiv \dot{a} / a$.

We consider a universe sourced by a perfect fluid with diagonal energy-momentum tensor, $T_{\mu \nu}=\operatorname{diag}\left(\rho, a^{2} p, a^{2} p, a^{2} p\right)$, where $\rho$ and $p$ denote the energy density and pressure 
of the fluid, respectively. We assume that during an infinitesimal time interval, $d t$, the area of the apparent horizon is effectively constant. The amount of energy, $d E$, that crosses the horizon during this interval can then be calculated by integrating the flux of the energy-momentum tensor through the horizon contracted with the (approximate) generator of the horizon, $k^{a}=(1,-H r)$. It follows that

$$
d E=-A T_{a b} k^{a} k^{b} d t=-\frac{4 \pi}{H^{2}}(\rho+p) d t=\frac{4 \pi}{3} \frac{d \rho}{H^{3}},
$$

where the condition for conservation of energy-momentum,

$$
\dot{\rho}+3 H(\rho+p)=0
$$

has been employed in the last equality.

It was recently shown that the apparent horizon of the FRW universe can be associated with Hawking radiation with a temperature given by Eq. (22) [18]. The first law of thermodynamics on the horizon, $d E=-T d S$, may therefore be expressed in the form

$$
d S=-\frac{8 \pi^{2}}{3} \frac{d \rho}{H^{4}}
$$

In the general case where the entropy of the apparent horizon is an arbitrary (differentiable) function of its area, $S=S\left(\pi / G H^{2}\right)$, Eq. (6) can be formally integrated to yield

$$
\rho=-\frac{3}{8 \pi^{2}} \int^{H} d H^{\prime 2}\left(H^{\prime 4} \frac{d S}{d H^{\prime 2}}\right)
$$

and Eq. (7) may be interpreted as a generalized Friedmann equation, where the constant of integration determines the value of the cosmological constant\&. When the entropy is given by the Bekenstein-Hawking entropy, the standard Friedmann equation, $\rho=3 H^{2} /(8 \pi G)$, is recovered [10]. When the entropy receives a logarithmic correction of the form (3), however, integration of Eq. (7) results in a modified Friedmann equation given by [17]

$$
H^{2}+\frac{\alpha G}{2 \pi} H^{4}=\frac{8 \pi G}{3} \rho .
$$

Eq. (8) can be solved quadratically to yield

$$
H^{2}=\frac{\pi}{\alpha G}\left[-1+\epsilon\left(1+\frac{16 \alpha G^{2}}{3} \rho\right)^{1 / 2}\right],
$$

where $\epsilon= \pm 1$.

$\S$ In this thermodynamic context, the cosmological constant is determined by the initial conditions [6]. We set it to zero in what follows. 


\section{Trace-Anomaly Friedmann Equation}

We now consider the effect of the conformal anomaly on the semi-classical gravitational field equations derived directly from quantum field theory. These equations can be expressed in the form

$$
R_{\mu \nu}-\frac{1}{2} R g_{\mu \nu}=8 \pi G\left\langle T_{\mu \nu}\right\rangle
$$

where $\left\langle T_{\mu \nu}\right\rangle$ represents the expectation value for the energy-momentum tensor of a large number of conformally invariant fields. For a general CFT, the total one-loop contribution to the trace of this tensor is given by the conformal anomaly [12]:

$$
g^{\mu \nu}\left\langle T_{\mu \nu}\right\rangle=c F-b G+d \nabla^{2} R
$$

where $F=C_{\mu \nu \lambda \kappa} C^{\mu \nu \lambda \kappa}$ is the square of the Weyl tensor and $G=R^{2}-4 R^{\mu \nu} R_{\mu \nu}+$ $R^{\mu \nu \lambda \kappa} R_{\mu \nu \lambda \kappa}$ is the topological Gauss-Bonnet invariant. The constant coefficients are determined by the field content of the CFT [13]:

$$
\begin{aligned}
& b=\frac{1}{360(4 \pi)^{2}}\left(n_{0}+11 n_{1 / 2}+62 n_{1}\right) \\
& c=\frac{1}{120(4 \pi)^{2}}\left(n_{0}+6 n_{1 / 2}+12 n_{1}\right) \\
& d=\frac{1}{180(4 \pi)^{2}}\left(n_{0}+6 n_{1 / 2}-18 n_{1}\right),
\end{aligned}
$$

where $n_{s}$ denotes the number of fields with spin $s$ and the spinor fields are Dirac fermions.

The coefficients $b$ and $c$ are independent of the renormalization scheme, whereas $d$ is scheme-dependent. Eq. (14) is the result derived from point-splitting or zeta-function regularization [13]. The same result is predicted by the AdS/CFT correspondence where the CFT is $\mathcal{N}=4, U(N)$ super-Yang-Mills theory [19]. We consider this CFT in what follows. Its field content is given by $n_{0}=6 N^{2}, n_{1 / 2}=2 N^{2}$ and $n_{1}=N^{2}$ in the large $N$ limit, which implies that

$$
b=c=\frac{N^{2}}{(8 \pi)^{2}}, \quad d=0 .
$$

Consequently, the trace of the one-loop energy-momentum tensor for this CFT reduces to

$$
\left\langle T_{\mu}^{\mu}\right\rangle=-24 b \frac{\ddot{a}}{a} \frac{\dot{a}^{2}}{a^{2}}
$$

on the conformally flat and spatially flat FRW background.

In deriving the modified Friedmann equation, it proves convenient to interpret the components of the quantum energy-momentum tensor in terms of an effective energy density and pressure, such that $\left\langle T_{00}\right\rangle \equiv \sigma$ and $\left\langle T_{i j}\right\rangle \equiv \sigma_{P} a^{2} \delta_{i j}$ [20, 21, 22]. The trace formula (16) can then be written as

$$
\sigma-3 \sigma_{P}=24 b \frac{\ddot{a}}{a} \frac{\dot{a}^{2}}{a^{2}},
$$


whereas the Bianchi identity reduces to the condition

$$
\dot{\sigma}+3 H\left(\sigma+\sigma_{P}\right)=0 .
$$

Combining Eqs. (17) and (18) to eliminate the effective pressure implies that the energy density generated by the trace anomaly satisfies the differential equation 22 ]

$$
\dot{\sigma}+4 H \sigma-24 b \frac{\ddot{a}}{a} \frac{\dot{a}^{3}}{a^{3}}=0
$$

and integrating Eq. (19) then yields the solution

$$
\sigma=\frac{\sigma_{0}}{a^{4}}+6 b H^{4},
$$

where $\sigma_{0}$ is an arbitrary integration constant. Consequently, the (00)-component of the gravitational field equations (10) may now be expressed in the form

$$
H^{2}-16 \pi G b H^{4}=\frac{8 \pi G}{3} \frac{\sigma_{0}}{a^{4}} .
$$

We conclude after comparison with Eq. (8), therefore, that the Friedmann equation for anomaly-driven cosmology can be derived from the first law of thermodynamics when the matter source is a conformally invariant classical fluid and the entropy of the apparent horizon is given by the logarithmically-corrected Bekenstein-Hawking relation (3). The correspondence is exact if the parameter $\alpha$ is proportional to the conformal anomaly:

$$
\alpha=-32 \pi^{2} b=-\frac{N^{2}}{2} .
$$

In the following Section, we discuss this thermodynamic interpretation of traceanomaly cosmology within the context of the Randall-Sundrum braneworld scenario and the AdS/CFT correspondence.

\section{Randall-Sundrum Braneworld and the AdS/CFT Correspondence}

Two of the most significant advances over the past decade towards a consistent theory of quantum gravity have been the AdS/CFT correspondence [23, 24, 25] and the RandallSundrum (RS) braneworld scenario [26]. In the RS scenario, our observable fourdimensional universe is interpreted as a co-dimension one-brane propagating in fivedimensional anti-de Sitter (AdS) space with a $Z_{2}$ reflection symmetry. From the point of view of a four-dimensional observer confined to the brane, the cosmic expansion arises due to the propagation of the brane through the bulk space. The effective fourdimensional Friedmann equation can be derived by applying the thin wall formalism of General Relativity to five dimensions and it can be shown that for a spatially flat world-volume

$$
H^{2}=\frac{8 \pi G}{3} \rho\left(1+\frac{\rho}{2 \lambda}\right),
$$

where $\rho$ represents the density of matter on the brane, $\lambda=3 G /\left(4 \pi G_{5}^{2}\right)$ denotes the brane tension and $G_{5}$ is the five-dimensional Newton constant [27, 28, 29]. (The 
tension is fine-tuned to this value to ensure that the brane world-volume reduces to Minkowski spacetime in the vacuum limit). Energy-momentum conservation of the four-dimensional matter fields, Eq. (5), follows as a direct consequence of the vanishing of the $(\mu 5)$-component of the five-dimensional Einstein tensor.

The AdS/CFT correspondence provides a realization of the holographic principle [30, 31] by relating semi-classical, $d$-dimensional gravity in AdS space to a quantum CFT located on the boundary of the spacetime [23, 24]. In particular, this correspondence implies that the RS model can be interpreted holographically as four-dimensional Einstein gravity coupled to a CFT with an ultra-violet cutoff. The CFT is two copies of the $\mathcal{N}=4 U(N)$ super-Yang-Mills theory, where $N^{2}=\pi \ell^{3} /\left(2 G_{5}\right)$ and $\ell=G_{5} / G$ is the AdS radius [32, 33]. It follows from Eq. (22), therefore, that the Friedmann equation for this model is given by Eq. (7), where $\alpha=-64 \pi^{2} b=-N^{2}$. Consequently, the dynamics of the holographic dual of the RS scenario can be described in terms of the first law of thermodynamics, such that

$$
\begin{aligned}
& d E=-T d S, \quad T=\frac{H}{2 \pi} \\
& S=\frac{\pi}{G H^{2}}+\alpha \ln \left(\frac{\pi}{G H^{2}}\right), \quad \alpha=-N^{2}=-\frac{\pi \ell^{3}}{2 G_{5}} .
\end{aligned}
$$

Since the four- and five-dimensional interpretations of the RS scenario are dual to one other, it should be possible to provide a thermodynamic derivation of the Friedmann equation (23) that followed directly from the five-dimensional gravitational field equations. To address this question, we now suppose that the first law of thermodynamics, Eqs. (24)-(25), can be applied to the apparent horizon of the worldvolume of the brane. We then proceed to express the first law in the form

$$
T d S_{\mathrm{BH}}=-d E-T d S_{\mathrm{cor}},
$$

where $S_{\text {cor }} \equiv \alpha \ln \left(\pi / G H^{2}\right)$ denotes the correction to the Bekenstein-Hawking entropy, $S_{\mathrm{BH}}$. It follows from Eq. (6) that Eq. (26) can be written as

$$
H^{4} d S_{\mathrm{BH}}=-\frac{8 \pi^{2}}{3} d \rho-H^{4} d S_{\text {cor }} .
$$

In the absence of the logarithmic correction, integration of Eq. (27) would lead to the standard Friedmann equation, $H^{2}=8 \pi G \rho / 3$. If we regard this as a 'zero-order' solution to Eq. (27) and substitute it back into the second term on the right-hand side, we may interpret this term as an explicit function of the energy density. It then follows that

$$
H^{4} d S_{\mathrm{BH}} \simeq-\frac{8 \pi^{2}}{3} d \rho-\frac{16 \pi^{3} \ell G_{5}}{9} d \rho^{2}
$$

when $\alpha$ is determined by the bulk quantities $\ell$ and $G_{5}$ given in the AdS/CFT relation (25). It is then straightforward to show that integration of Eq. (28) leads directly to the RS Friedmann equation (23). In this sense, the RS scenario may be viewed as the low-energy/high-entropy limit of the anomaly-driven cosmology. This is consistent with Taylor expanding Eq. (9) to quadratic order in the energy density [22, 34]. 


\section{Conclusion}

Cosmology driven by the trace anomaly of conformally invariant matter fields represents an important framework for investigating the evolution of the very early universe. The correspondence highlighted in the present work allows us to interpret the effect of the anomaly from a thermodynamic perspective as a logarithmic correction to the Bekenstein-Hawking entropy associated with the apparent horizon of the spatially flat FRW universe. The Friedmann equation derived by applying the first law of thermodynamics to the apparent horizon with such a corrected entropy has the same form as that derived directly from field-theoretic techniques. This is interesting given that a similar logarithmic correction to the Bekenstein-Hawking entropy is generated by one-loop effects near the event horizon of a Schwarzschild black hole [14]. In both the cosmological and black hole backgrounds, the correction is determined by the number of zero-mass fields: for the FRW universe, $\alpha \propto-b$, whereas $\alpha \propto(c-b)$ for the Schwarzschild black hole [14].

We have focused on the trace anomaly of $\mathcal{N}=4 U(N)$ super-Yang-Mills theory and our conclusions rely on the condition that the anomaly coefficient defined in Eq. (14) vanishes. A non-zero value would lead to terms involving higher derivatives of the scale factor in the expression for the effective energy density of the anomaly, Eq. (20). Nonetheless, we expect this correspondence between thermodynamics and gravity to hold for other theories where $d \neq 0$. This follows since the value of this parameter can always be renormalized to zero by introducing a finite local counterterm (proportional to $R^{2}$ ) into the non-local action.

In conclusion, therefore, the present work provides a further example of how thermodynamics emerges from a combination of quantum physics and gravitation.

\section{References}

[1] Bekenstein J D, Black holes and entropy, 1973 Phys. Rev. D 7 2333-2346

[2] Hawking S W, Particle creation by black holes, 1975 Commun. Math. Phys. 4343

[3] Gibbons G W and Hawking S W, Cosmological event horizons, thermodynamics, and particle creation, 1977 Phys. Rev. D 15 2738-2751

[4] Jacobson T, Thermodynamics of space-time: The Einstein equation of state, 1995 Phys. Rev. Lett. 751260 gr-qc/9504004

[5] Frolov A V and Kofman L, Inflation and de Sitter thermodynamics, 2003 JCAP 0305009 hep-th/0212327

[6] Danielsson U H, Transplanckian energy production and slow-roll inflation, 2005 Phys. Rev. D71 023516 hep-th/0411172

[7] Bousso R, Cosmology and the S-matrix, 2005 Phys. Rev. D71 064024 hep-th/0412197

[8] Wang P, Horizon entropy in modified gravity, 2005 Phys. Rev. D72 024030 gr-qc/0507034

[9] Gong Y and Wang A, The Friedmann equations and thermodynamics of apparent horizons, 2007 Phys. Rev. Lett. 992113010704.0793.

[10] Cai R G and Kim S P, First law of thermodynamics and Friedmann equations of FriedmannRobertson-Walker universe, 2005 JHEP 02050 hep-th/0501055

[11] Wu S F, Wang B and Yang G H, Thermodynamics on the apparent horizon in generalized gravity theories, 2008 Nucl. Phys. B799 330-344 0711.1209. 
[12] Duff M J, Twenty years of the Weyl anomaly, 1994 Class. Quant. Grav. 11 1387-1404 hep-th/9308075

[13] Birrell N D and Davies P C W, Quantum Field Theory in Curved Spacetime, 1982 Cambridge University Press

[14] Fursaev D V, Temperature and entropy of a quantum black hole and conformal anomaly, 1995 Phys. Rev. D51 5352-5355 hep-th/9412161

[15] Banerjee R and Majhi B R, Quantum tunneling beyond semi-classical approximation, 2008 JHEP 060950805.2220

[16] Banerjee R and Majhi B R, Quantum tunneling, trace anomaly and effective metric, 2008 0808.3688

[17] Cai R G, Cao L M and Hu Y P, Corrected entropy-area relation and modified Friedmann equations, 2008 JHEP 080900807.1232

[18] Cai R G, Cao L M and Hu Y P, Hawking radiation of apparent horizon in a FRW Universe, 2008 0809.1554

[19] Henningson $\mathrm{M}$ and Skenderis K, The holographic Weyl anomaly, 1998 JHEP 07023 hep-th/9806087

[20] Fischetti M V, Hartle J B and Hu B L, Quantum effects in the early universe I: Influence of trace anomalies on homogeneous, isotropic, classical geometries, 1979 Phys. Rev. D 20 1757-1771

[21] Hawking S W, Hertog T and Reall H S, Trace anomaly driven inflation, 2001 Phys. Rev. D63 083504 hep-th/0010232

[22] Kiritsis E, Holography and brane-bulk energy exchange, 2005 JCAP 0510014 hep-th/0504219

[23] Maldacena $\mathrm{J} \mathrm{M}$, The large $N$ limit of superconformal field theories and supergravity, 1998 Adv. Theor. Math. Phys. 2 231-252 hep-th/9711200

[24] Witten E, Anti-de Sitter space and holography, 1998 Adv. Theor. Math. Phys. 2 253-291 hep-th/9802150

[25] Gubser S S, Klebanov I R and Polyakov A M, Gauge theory correlators from non-critical string theory, 1998 Phys. Lett. B428 105-114 hep-th/9802109

[26] Randall L and Sundrum R, An alternative to compactification, 1999 Phys. Rev. Lett. 83 4690-4693 hep-th/9906064

[27] Binetruy P, Deffayet C, Ellwanger U and Langlois D, Brane cosmological evolution in a bulk with cosmological constant, 2000 Phys. Lett. B477 285-291 hep-th/9910219

[28] Cline J M, Grojean C and Servant G, Cosmological expansion in the presence of extra dimensions, 1999 Phys. Rev. Lett. 834245 hep-ph/9906523

[29] Shiromizu T, Maeda K and Sasaki M, The Einstein equations on the 3-brane world, 2000 Phys. Rev. D62 024012 gr-qc/9910076

[30] 't Hooft G, Dimensional reduction in quantum gravity, 1993 gr-qc/9310026

[31] Susskind L, The world as a hologram, 1995 J. Math. Phys. 36 6377-6396 hep-th/9409089

[32] Gubser S S, AdS/CFT and gravity, 2001 Phys. Rev. D63 084017 hep-th/9912001

[33] Hawking S W, Hertog T and Reall H S, Brane new world, 2000 Phys. Rev. D62 043501 hep-th/0003052

[34] Barvinsky A O, Deffayet C and Kamenshchik A Y, Anomaly-driven cosmology: big boost scenario and AdS/CFT correspondence, 2008 JCAP 0805020 [0801.2063. 